\title{
Discovery of remote star clusters in the halo of the Irregular galaxy NGC 6822
}

\author{
Narae Hwang ${ }^{1}$, Myung Gyoon Lee ${ }^{1}$, Jong Chul Lee ${ }^{1}$, Won-Kee Park ${ }^{1}$, \\ Hong Soo Park ${ }^{1}$, Jang-Hyun Park ${ }^{2}$, Sangmo Tony Sohn ${ }^{2}$, \\ Sang-Gak Lee ${ }^{1}$, Hyung Mok Lee ${ }^{1}$, Mun-Suk Chun ${ }^{3}$, \\ Young-Wook Lee ${ }^{3}$, Young-Jong Sohn ${ }^{3}$, In-Soo Yuk ${ }^{1,2}$, \\ Sang Chul Kim ${ }^{1,2}$, Ho-Il Kim ${ }^{2}$ and Wonyong Han ${ }^{2}$ \\ ${ }^{1}$ Astronomy Program, SEES, Seoul National University, Seoul 151-742, Korea \\ email: nhwang@astro.snu.ac.kr \\ ${ }^{2}$ Korea Astronomy \& Space Science Institute, 61-1 Whaam Yuseong, Daejeon 305-348, Korea \\ ${ }^{3}$ Center for Space Astrophysics, Yonsei University, Seoul 120-179, Korea
}

\begin{abstract}
We report the discovery of three new star clusters in the halo of the Local Group dwarf irregular galaxy NGC 6822. These clusters were found in the deep images taken with the MegaPrime at the CFHT, covering a total field of $2 \mathrm{deg} \times 2 \mathrm{deg}$. The most remote cluster is found to be located as far as 79 arcmin away from the center of NGC 6822 . This distance is several times larger than the size of the region in NGC 6822 where star clusters were previously found. Morphological structures of the clusters and color-magnitude diagrams of the resolved stars in the clusters show that at least two of these clusters are proabably old globular clusters.
\end{abstract}

Keywords. globular clusters: general, galaxies: dwarf, galaxies: individual (NGC 6822)

\section{Introduction}

Star clusters in the dwarf irregular galaxy NGC 6822 have been first reported by Hubble (1925) and systematically investigated by Hodge (1977). The most recent study by Krienke \& Hodge (2004) used the available HST Archive data and cataloged all the star clusters identified including one genuine globular cluster Hubble VII. However, no star cluster survey in the outer halo of NGC 6822 has ever been tried before, although the existence of a rather large stellar halo around the galaxy was suggested by Letarte et al. (2002) from their Carbon star survey.

\section{Discovery}

Visual inspection of the wide field survey data around NGC 6822 has revealed three new star clusters. The locations and morphologies of these clusters are shown in Figure 1. From morphological and photometric studies, two clusters, SC1 and SC2, are regarded genuine old globular clusters with ages more than 3 Gyrs (see Hwang et al. 2005 for details). One noteworthy point is that the new star clusters are distributed to the very remote places (Left panel of Figure 1). The projected distance from the NGC 6822 center to SC1, the most remote cluster, is about $12 \mathrm{kpc}$. For comparison, NGC 1841, the outermost star cluster in the LMC, is located at about $13 \mathrm{kpc}$ from the LMC center. Another important point in the images is that all three clusters are extended and are clearly resolved into stars, whereas Hubble VII is not resolved at all in our data (Right panel of Figure 1). Further investigation shows that the half-light radii of these new clusters are larger than $10 \mathrm{pc}$ and even larger than $20 \mathrm{pc}$ for SC1. 

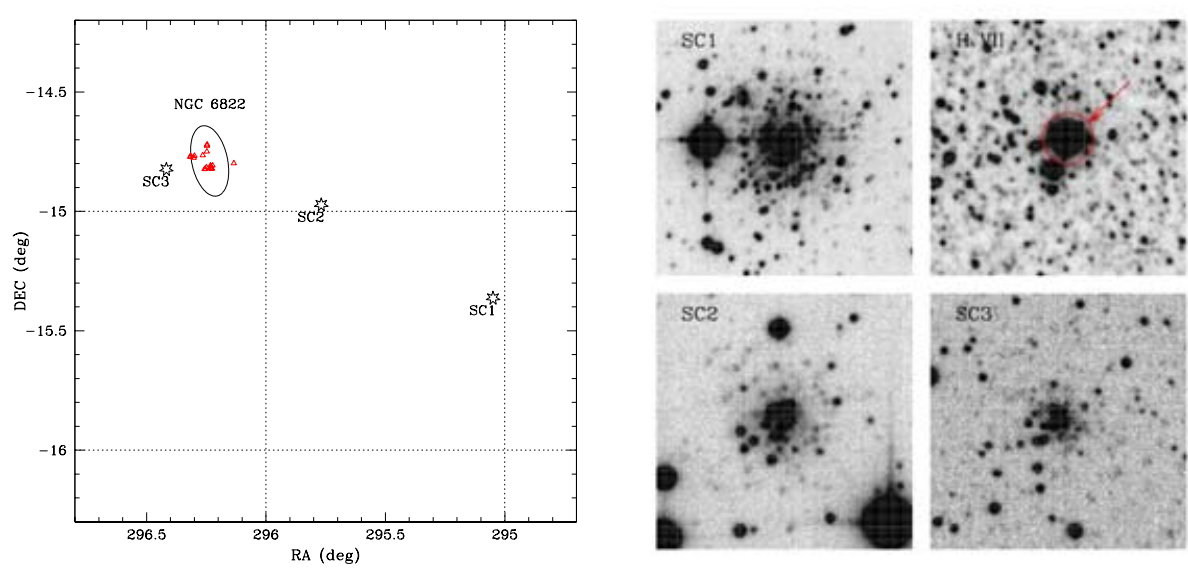

Figure 1. Left: Locations of the new star clusters in NGC 6822 (stellar symbols). The SC1 is about $12 \mathrm{kpc}$ away from the galaxy center. Marks (triangles) inside and around the ellipse are previously known star clusters. Right: Sloan $i$ band images of the three new star clusters and a known globular cluster Hubble VII in NGC 6822 (upper right; marked by an arrow). Note the resolved member stars of new star clusters. Each image is $37^{\prime \prime} \times 37^{\prime \prime}$ wide.

\section{Implications}

The existence of the newly discovered star clusters suggests that the underlying halo has a different structure from a giant HI disk-like cloud which is extended along NW-SE direction (de Blok \& Walter 2000). These clusters also provide a proof that the halo of NGC 6822 is quite larger than previously expected (see Lee \& Hwang 2005 in this volume).

The extended structures of these new clusters are very unusual features. The SC1, among these, is found to be as extended as new star clusters recently discovered in the halo of M31 (Huxor et al. 2005; Lee et al. 2005). The formation mechanism of these extended star clusters, including the correlation with evolutionary history of the host galaxies, is not clearly understood yet, requiring further studies.

\section{Acknowledgements}

N.Hwang was supported in part by the BK21 program. M.G.Lee was supported in part by the ABRL(R14-2002-058-010000-0).

\section{References}

de Blok, W.J.G. \& Walter, F. 2000, ApJ 537, L95

Hodge, P.W. 1977, ApJS 33, 69

Hubble, E.P. 1925, ApJ 62, 409

Huxor, A.P., Tanvir, N.R., Irwin, M.J., Ibata, R., Collett, J.L., Ferguson, A.M.N., Bridges, T. \& Lewis, G.F. 2005, MNRAS in press (astro-ph/0412223)

Hwang, N. et al. 2005, in preparation

Krienke, K. \& Hodge, P. 2004, PASP 116, 497

Lee, M.G. \& Hwang, N. 2005, this proceedings

Lee, M.G. et al. 2005, in preparation

Letarte, B., Demers, S., Battinelli, P. \& Kunkel, W.E. 2002, AJ 123, 832 